\title{
LV-PP-3-6
}

\section{Surgical Resection of Huge Hepatocellular Carcinoma. Prognostic Factors and Outcomes in a Mexican Cohort Study}

\author{
Carlos FLOREZ-ZORRILLA ${ }^{* 1,2,3}$, Karina MENDOZA-GALLO \\ 'Division of Hepatobiliary and Pancreatic Surgery and Liver Transplant, Hospital Puerta de Hierro Andares, Guadalajara, Mexico \\ ${ }^{2}$ Division of Hepatobiliary and Pancreatic Surgery and Liver Transplant, Hospital Ángeles Lomas, Ciudad de México, Mexico \\ ${ }^{3}$ Division of Hepatobiliary and Pancreatic Surgery and LIver Transplant, Centro Médico ABC, Ciudad de México, Mexico
}

Introduction: Surgery is the best choice for curative treatment for hepatocellular carcinoma (HCC), that includes liver resection (LR) or liver transplantation (LT). Tumors larger than $10 \mathrm{~cm}$ are considered huge HCC. Huge HCC doesn't fit any criteria for LT. Although surgical resection may be associated with higher morbidity it's still the best chance of survival in this subgroup of patients. The purpose of our study is to analyze prognostic factors and outcomes in a Mexican population.

Methods: We analyzed patients who underwent LR for huge HCC between January 2015 to January 2021. Prognostic factors included in the study were age, HCC size, serum alpha fetoprotein (AFP) and vascular invasion. Only patients with Child-Pugh grade A in absence of extrahepatic disease was offered major LR. The future liver remnant volume was calculated by CT scan volumetry. Morbidity, disease free-survival and overall survival was evaluated.

Results: Fifty two patients underwent major LR: male 30 (57.6\%) and female 22 (42.3\%). The median age was 65 years (range, 25-76 years), median HCC size was $16 \mathrm{~cm}$ (range, 14-45 cm), median AFP was $85 \mathrm{ng} / \mathrm{mL}$ (range, 18-19,500 ng/mL). Lymphovascular invasion was found in $58 \%$ of liver specimens. The overall morbidity was $12 \%$ and perioperative mortality was seen in 1 (1.95\%) due to heart failure. The overall 1-year and 3-year survival were $88.4 \%$ and $51.9 \%$ respectively,

Conclusions: Factors of adverse outcome after major LR in patients with huge HCC were the presence of lymphovascular invasion, AFP $>1,000 \mathrm{ng} / \mathrm{mL}$, and age $>65$ years. 